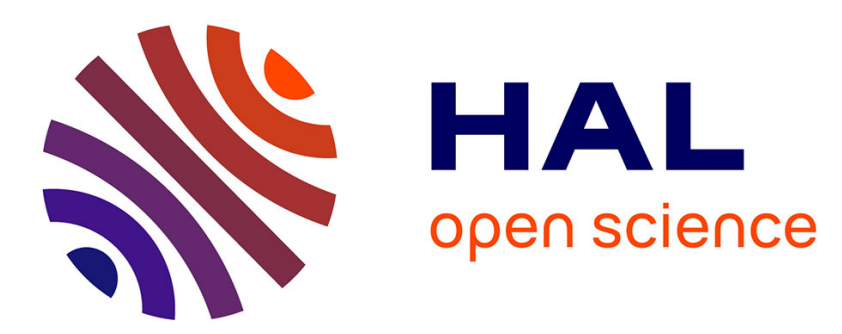

\title{
Fate of nanoplastics in the environment: Implication of the cigarette butts
}

Hind El Hadri, Jesus Maza Lisa, Julien Gigault, Stephanie Reynaud, Bruno Grassl

\section{- To cite this version:}

Hind El Hadri, Jesus Maza Lisa, Julien Gigault, Stephanie Reynaud, Bruno Grassl. Fate of nanoplastics in the environment: Implication of the cigarette butts. Environmental Pollution, 2021, 268, pp.115170. 10.1016/j.envpol.2020.115170 . insu-02899294

\section{HAL Id: insu-02899294 https://hal-insu.archives-ouvertes.fr/insu-02899294}

Submitted on 15 Jul 2020

HAL is a multi-disciplinary open access archive for the deposit and dissemination of scientific research documents, whether they are published or not. The documents may come from teaching and research institutions in France or abroad, or from public or private research centers.
L'archive ouverte pluridisciplinaire HAL, est destinée au dépôt et à la diffusion de documents scientifiques de niveau recherche, publiés ou non, émanant des établissements d'enseignement et de recherche français ou étrangers, des laboratoires publics ou privés. 


\section{Journal Pre-proof}

Fate of nanoplastics in the environment: Implication of the cigarette butts

Hind El Hadri, Jesus Maza Lisa, Julien Gigault, Stéphanie Reynaud, Bruno GrassI

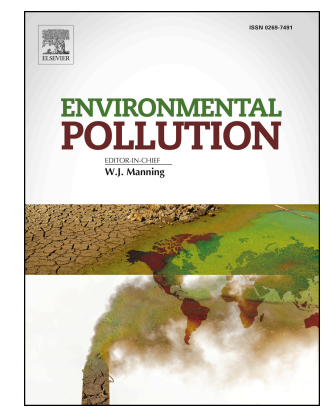

PII: S0269-7491(20)32958-4

DOI: $\quad$ https://doi.org/10.1016/j.envpol.2020.115170

Reference: ENPO 115170

To appear in: Environmental Pollution

Received Date: 20 April 2020

Revised Date: 30 June 2020

Accepted Date: 2 July 2020

Please cite this article as: El Hadri, H., Lisa, J.M., Gigault, J., Reynaud, Sté., Grassl, B., Fate of nanoplastics in the environment: Implication of the cigarette butts, Environmental Pollution (2020), doi: https://doi.org/10.1016/j.envpol.2020.115170.

This is a PDF file of an article that has undergone enhancements after acceptance, such as the addition of a cover page and metadata, and formatting for readability, but it is not yet the definitive version of record. This version will undergo additional copyediting, typesetting and review before it is published in its final form, but we are providing this version to give early visibility of the article. Please note that, during the production process, errors may be discovered which could affect the content, and all legal disclaimers that apply to the journal pertain.

() 2020 Published by Elsevier Ltd. 
Fate of nanoplastics in the environment: implication of the cigarette butts

Hind El Hadri, Jesus Maza Lisa, Julien Gigault, Stéphanie Reynaud, Bruno Grassl

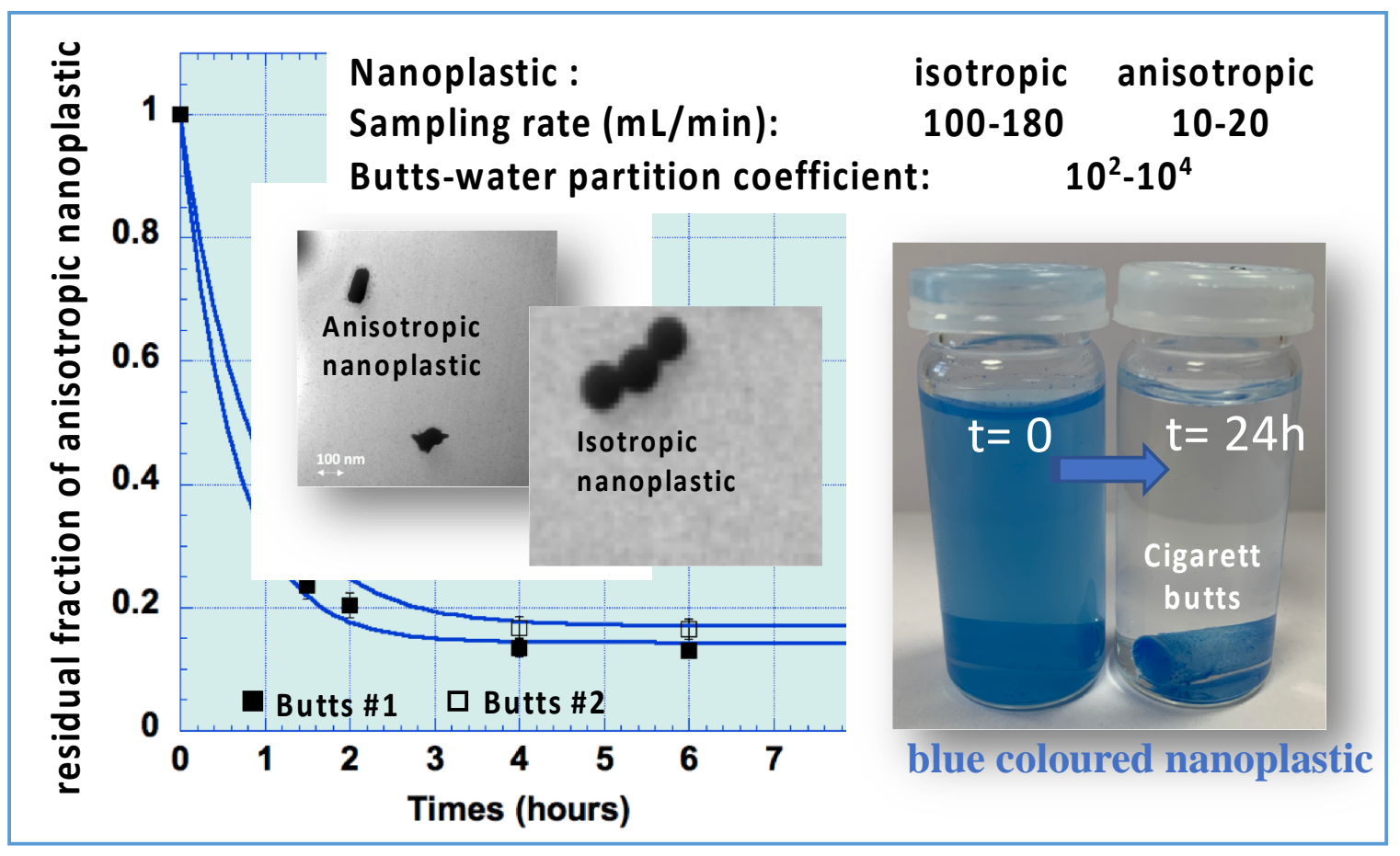


a. CNRS/ Univ Pau \& Pays Adour/ E2S UPPA, Institut des sciences analytiques et de physicochimie pour l'environnement et les matériaux, UMR 5254, 64000, Pau, France

b. Géosciences Rennes, UMR 6118, CNRS - Université de Rennes 1, Av. Général Leclerc, Campus de Beaulieu, 35000 Rennes France

*Corresponding author: stephanie.reynaud@univ-pau.fr

Abstract: Fate, transport and accumulation of nanoplastics have attracted considerable attention in the past few years. While actual researches have been focused on nanoplastics dispersed or aggregated in different environmental system, no study have been focused on the possibility that nanoplastics are cotransported with other natural or anthropogenic materials. Therefore, the large quantity of debris released in the environment, such as cigarette butts (CGB), could be part of the nanoplastics fate and behavior. Here we show the considerable sorption capacities of cigarette filters for nanoplastics. To address this topic, we chose polystyrene-based nanoplastics with similar state of charge (according to the physico-chemical characteristic of the zeta potential -45 to $-40 \mathrm{mV}$ ) but with different sizes (50$800 \mathrm{~nm})$ and morphologies. A kinetic approach to sorption in fresh water $\left(\mathrm{pH}=8.05 ; 179.5 \mu \mathrm{Sm}^{-1}\right)$ at room temperature was carried out by means of the flow field flow analysis method (AF4) to determine the partition coefficients and water sampling rates between nanoplastics and cigarette butts. Using different models of, more or less environmentally relevant, nanoplastics (NPTs) and adequate analytical strategies, we found partition coefficients between the NPTs and CGBs ranged from $10^{2}$ to $10^{4}$ in freshwater conditions. We demonstrated that the physical features of the NPTs (size and morphology) 
24 seems to be mostly retained in the CGBs after longer equilibration time. This result shows the 25 importance of the NPTs features on the mechanisms governing their transfer and fate in the 26 environment through environmental matrices, especially when other materials are involved. We 27 anticipate our work to be a starting point for investigating the co-transport of NPTs with other materials 28 present in the environment (natural and anthropogenic).

30 Keywords: nanoplastic, cigarette butts, sorption.

\section{Introduction}

33 Plastic pollution has attracted considerable attention from the public sector in recent years.

34 While a large majority of the relevant research has been performed on microsized plastic 35 particles, only a few groups of researchers focused their work on nanoscale plastics (Li et al., 36 2020; Shen et al., 2019; Wagner and Reemtsma, 2019). It was now admitted that the 37 fragmentation of plastic particles releases a considerable number of nanoplastics (NPTs) (Gigault 38 et al., 2016; Hartmann et al., 2019; Lambert and Wagner, 2016a, 2016b). Similar to other classic 39 nanoparticles, NPTs may interact with various species, whether naturally present or 40 (un)intentionally released within the environment. These interactions can enhance NPT 41 transport through different natural interfaces (soil-freshwater-estuarine system-ocean) or 42 promote their accumulation in specific areas (Alimi et al., 2018; da Costa et al., 2016; Dong et al., 43 2019). 
Among all the types of debris found in the environment, cigarette butts (CGBs) are one of the most predominant, and worldwide cigarette consumption is estimated to reach approximately 9 trillion by 2025 (Araújo and Costa, 2019; Mackay et al., 2002). It is estimated that more than 750 000 metric tons of CGBs end up as litter worldwide per year, which corresponds to approximately $4.510^{12}$ CGB units released into our environment each year (Novotny and Slaughter, 2014). Because CGBs are composed of cellulose acetate, they can interact with several organic and metallic pollutants in the environment (Chevalier et al., 2018). Pu et al. recently demonstrated that CGB based-materials act as a powerful Uranium sorbent (106 $\mathrm{mg} \mathrm{g}^{-1}$ ) (Pu et al., 2019). In air, CGBs were demonstrated to sorb and release particles with different size distribution (Hengstberger and Stark, 2009). In aqueous media, due to the large quantity of CGBs and nanoplastics released, co-transport of NPTs and other contaminants with CGBs have to be investigated for environmental and health perspectives. Indeed, NPT-CGB interaction and synergy could complicate the fate and impact of both of these emergent pollutants, whose behaviour is already challenging.

The main objective of this work is to investigate the sorption capacities of NPTs on cigarette filters to evaluate the general problem of the co-transport possibility of these nanoplastics in the environment. In this work, different kinds of nanoplastics were chosen. The first type of NPTs tested is the one commonly used in environmental studies on the impacts of NPTs on aquatic organisms and the transfer and environmental fate of NPTs. These NPTs are monodisperse and spherical (Pessoni et al., 2019). On the other hand, the second type of NPTs was chosen to be anisotropic and was obtained by mechanical fragmentation as described elsewhere (Davranche et al., 2019; El Hadri et al., 2020; Gillibert et al., 2019). Both of these NPT series are identical in terms of chemical composition (polystyrene base) and have similar surface functionality, as revealed by their similar values of the zeta-potential physico-chemical parameter. A kinetic 
approach to analyze NPT sorption on unused cigarette filters was performed, the partition coefficients and mass transfer coefficients in fresh water have been evaluated.

\section{Materials and Methods}

Reagents. In this work, different nanoplastics have been studied: (i) commercial polystyrene latex NPTs, called PSLs (certified carboxylated PSL NPTs), which are often used to study the effects of nanoplastics on aquatic organisms, (Pikuda et al., 2018) and (ii) fragmented polystyrene NPTs, called NPTf, produced via a top-down method based on mechanical degradation of primary polystyrene microplastics and recently described elsewhere (El Hadri et al., 2020). The sodium nitrate used to formulate the mobile phase for the flow field-flow fractionation (AF4) was purchased from Sigma-Aldrich (Darmstadt, Germany). Polyethersulfone (PES) filters $(0.1 \mu \mathrm{m})$, purchased from VWR (Fontenay-sous-Bois, France), were used to remove particles from the mobile phase. All the solutions were prepared with ultrapure deionized water (18.2 $\mathrm{M} \Omega \mathrm{cm}$, at $25^{\circ} \mathrm{C}$ ) obtained from a Milli-Q system (Millipore, Molsheim, France). The polystyrene latex (PSL) carboxylated beads with hydrodynamic diameters $\left(d_{H}\right)$ of $50 \mathrm{~nm}, 180 \mathrm{~nm}$ and $500 \mathrm{~nm}$ and blue-dyed PSLs $(200 \mathrm{~nm}$ ) were purchased from Polysciences (Hirschberg an der Bergstraße, Germany). The pellets of polystyrene (PS) were purchased from Goodfellow (Lille, France). The freshwater was sampled from the Gave of Pau at the location of the Laroin Bridge (GPS coordinates: 43.305443, -0.436205) and presented a pH of 8.05 and a conductivity of 179.5 $\mu \mathrm{S} \mathrm{cm}{ }^{-1}$ at $23.1^{\circ} \mathrm{C}$.

Nanoplastic preparation. The protocol described elsewhere has been reproduced (El Hadri et al., 2020). Briefly, the fragmentation for NPT production was performed using a planetary ball mill with $5 \mathrm{~mm}$ zirconium oxide balls: 120 minutes at $450 \mathrm{rpm}$ processes in 4 steps (10 cycles of 3 
minutes of grinding and 6 minutes of a pause). A total organic carbon instrument (Shimadzu, Kyoto, Japan) was used to determine the concentration of the dispersion. Characterization: 59 $\mathrm{mg} \mathrm{kg}^{-1}$ in concentration, DLS $\left(d_{H}=310 \pm 10 \mathrm{~nm}\right)$, zeta potential in pure water $(\zeta=-42.5 \pm 2.0$ $\mathrm{mV}$ ), and transmission electronic microscopy (TEM) images given in supporting information (SI).

Cigarette filter specifics. Concerning the filters, two brands were used: "filter tip" from Rizla and "filter tub" from Gizeh, named R and G, respectively. These filters are both composed of hydrophilic cellulose acetate. For $\mathrm{R}$ and $\mathrm{G}$, length $=16.0$ and $15.0 \mathrm{~mm}$, diameter $=6.0$ and 8.0 $\mathrm{mm}$, dry mass $=59.5$ and $101.3 \mathrm{mg}$, dry mass per volume (density) $=131$ and $134 \mathrm{mg} / \mathrm{cm}^{3}$, wet mass $=412$ and $828 \mathrm{mg}$, respectively. All the values are based on an average of measurements on 20 filters.

Instruments. The AF4 system Eclipse 3+ AF4 instrument (Wyatt Technology, Dernbach, Germany) with a 250- $\mu$ m channel thickness and a 10-kDa PES membrane, a 1200 series highperformance liquid chromatography pump (Agilent Technologies, Les Ulis, France) equipped with a UV-vis absorption detector (1200 series, Agilent Technologies, Les Ulis, France) and a multiangle laser light scattering (MALS) detector (DAWN HELEOS, Wyatt Technology) has been used. Data from the detectors were collected and analysed by ASTRA version 6 (Wyatt Technology). The AF4 conditions for characterizing nanoplastics were previously optimized and published (Gigault et al., 2017).

Kinetic experiments: Ten millilitres of dispersing media with $5 \mathrm{mg} \mathrm{L}^{-1}$ NPTs was placed in a glass vial, and then one CGB was introduced into the solution before gently stirring (orbital stirring, 100 RPM) the solution for a defined period of time. It should be noted that the CGB was 
previously soaked in water to swell it. At time $t, 2 \mathrm{~mL}$ of the medium was collected, filtered to

$1.2 \mu \mathrm{m}$ and injected into the AF4 apparatus. Blank preparations with no filters or NPTs were also realized under the same conditions. Blanks with NPTs but without CGBs had a UV signal that corresponded to $t=0$. Blanks with CGBs but without NPTs had no UV or MALS signals. All the

Use of the UV peak area $\left(A_{w}\right)$ of the NPT dispersions from A4F-UV-SLS as a function of the exposure time $t$ allows to determine the total concentration of NPT in each solution $\left(C_{w}\right)$ from the residual fraction $f$. The latter is defined as follows: $f=A_{w} / A_{w 0}=C_{w} / C_{w 0}$, where $A_{w o}$ and $C_{w o}$ are the peak area and the concentration of the initial NPT sample, respectively, based on the mass in the aqueous medium. Experiments with known concentrations of PSLs (50, 180 and 500 $\mathrm{nm}$ ) yielded $f=0.009 \pm 0.001$ and $f=0.003 \pm 0.001$ as the limits of quantification (LQ) and detection (LD), respectively. Both types of NPT were mixed with CGBs in aqueous media, and withdrawals of the supernatant were taken and analysed by an analytical method to determine the potential interaction between CGBs and PSLs or NPTf.

Sorption model: The process governing the sorption of the NPT analyte is the overall result of the competition between sorption and release. Fick's diffusion law quantifies this sorption process, describes the exchange kinetics between cigarette butts and the aqueous phase, and may be expressed as follows:

$$
\frac{d C_{s}}{d t}=\frac{R_{s}}{V_{s}}\left(C_{w}-\frac{C_{s}}{K_{S w}}\right)
$$
equation 1

where $C_{s}$ and $C_{w}$ are the NPT concentrations at time $t$ in the CGBs and in water, respectively; $V_{s}$ is the CGB volume; $R_{s}$ is the water sampling rate through the CGBs, which is linearly proportional to the exchange surface of the filter; $K_{S W}$ is the CGB-water partition coefficient defined as the 
nanoplastics ratio in CGB and water $\left(C_{C G B} / C_{w}\right)$; and the terms $R_{S} C_{w} / V_{S}$ and $R_{S} C_{S} / V_{S} K_{S W}$ are the

expressions for sorption and release, respectively. The experiments correspond to a static exposure design in which the NPT concentration decreases with the exposure time. The solution of equation (1) is:

$$
f=\frac{C_{w}}{C_{w 0}}=\frac{\left\{1+\frac{K_{s w} V_{S}}{V_{w}} \exp \left[-\left(1+\frac{K_{S w} V_{S}}{V_{w}}\right) \frac{R_{S} t}{K_{S w} V_{S}}\right]\right\}}{1+\frac{K_{S w} V_{S}}{V_{w}}}
$$

equation 2

where $V_{w}$ is the volume of the aqueous solution. In this model, for a simple case of exposure, it is assumed that (i) the rate of mass transfer to CGBs (receiving phase) is linearly proportional to the difference between the chemical activity of the contaminant (NPTs) in the water phase and that in CGBs; (ii) an equilibrium exists at the interface; and (iii) molecular diffusion is the predominant transport mechanism within CGBs with a diffusion coefficient that is time- and concentration-independent.

\section{Results and discussion}

Sample characterization. The hydrodynamic diameters $\left(d_{H}\right)$ determined by dynamic light scattering (DLS) for the three PSLs batches are $50 \pm 2,198 \pm 10$, and $502 \pm 30 \mathrm{~nm}$, respectively, with $\zeta$ values in the range of -45 to $-40 \mathrm{mV}$. NPT $f$ presents an average $d_{H}$ of $(310 \pm 10) \mathrm{nm}$, which is in the middle of the range of sizes for the PSL samples, and a $\zeta$ value in pure water of $(-42.5 \pm$ 2.0) $\mathrm{mV}$, which is in the range of values for the PSL samples. NPTf is polydisperse and polymorphic (Figure S1); indeed, the particle shape significantly deviates from those of the spherical monodisperse PSLs. The size distributions of both types of NPTs were compared by AF4-UV-SLS (Figure 1). The NPT $f$ signal ranges from 13 to $16 \mathrm{~mL}$, corresponding to sizes ranging from $180 \mathrm{~nm}$ to $800 \mathrm{~nm}$ in equivalent hydrodynamic diameter (based on retention volume). The 

shape/structure of NPT $f$ and has been explained elsewhere (Brewer and Striegel, 2011).

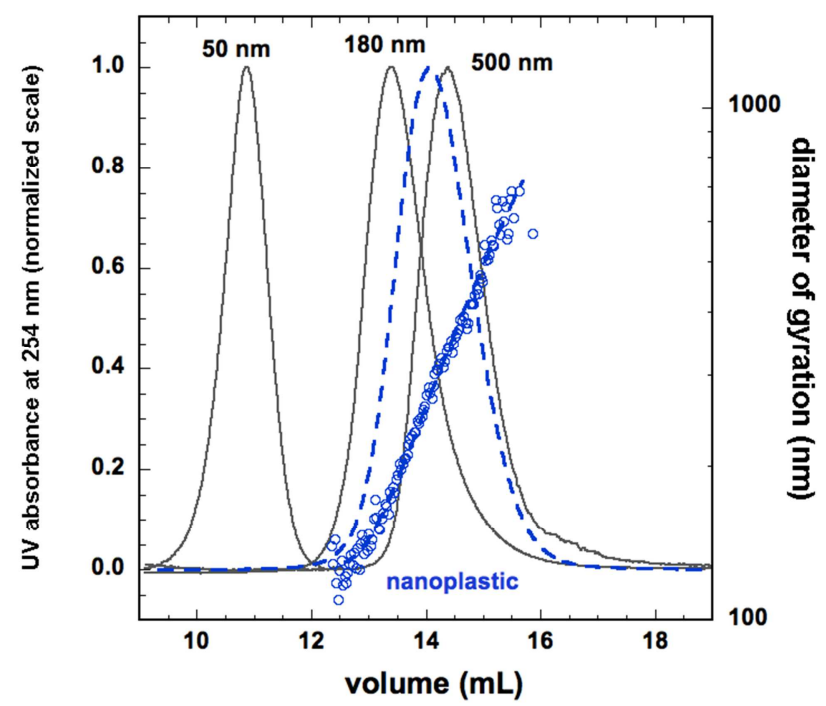

Figure 1. AF4-UV fractograms obtained for PSLs (50 nm, 180 and $500 \mathrm{~nm}$ ) solutions, and NPTf solutions (blue dashed line) with gyration diameter variation given by the MALS detector (blue open symbol).

Particle influence. Figure 2 shows the kinetics of the residual fraction $f$ for PSLs (Figure $2 a$ ) and NPT $f$ (Figure $2 \mathrm{~b}$ ) in the presence of CGB-R and CGB-G in freshwater at room temperature and under gentle agitation. It is worth mentioning that freshwater has been chosen because of the lack of aggregation of NPTs in this medium, as opposed of the quick aggregation phenomenon observed in a saline environment (critical coagulation concentration of approximatively $25 \mathrm{~g} / \mathrm{L}$ in $\mathrm{NaCl}$ ) (Schmitt et al., 2014). Within Figure 2, each time corresponds to one experiment performed under the same triplicate conditions: one prehydrated filter at size equilibrium left in $10 \mathrm{~mL}$ of a $5 \mathrm{ppm}$ nanoplastic solution. Under these conditions, the PSL residual fraction $(f)$ decreases rapidly within 0.5 hour to reach an equilibrium after 1 hour, and this behaviour seems

178 to be dependent on the PSL characteristics: the 500-nm PSL concentration decreases to a 179 residual fraction of $f=0.1$, while the $50-\mathrm{nm}$ PSL concentration decreases to $f=0.01$. However, 
for the experimental conditions tested, no correlation was found between the size of the PSL and the corresponding sorption kinetics, kinetic equilibrium is reached after 0.5 hours regardless of the PSL size. A similar experiment was then performed by mixing both CGB-R and CGB-G with an NPT $f$ sample. Compared to the previous results, the residual fractions of the overall NPTf sample at kinetic equilibrium are $f=0.12$ and $f=0.15$ for CGB-R and CGB-G, respectively. These results, obtained for an NPT $f$ sample exhibiting a continuum of sizes from $100 \mathrm{~nm}$ to $800 \mathrm{~nm}$ (see Figure 1), are in accordance with the previous results obtained for PSLs of discrete sizes. hour regardless of the size of the PSL samples (see Figure 2a). Since NPTs have similar chemical compositions and hydrodynamic size ranges, this difference could be attributed to the physical properties of the nanoplastic batches, i.e., PSLs versus NPT $f$. The longer equilibrium time of the NPT $f$ could be attributed to the difference in shape/structure as characterized by TEM (Figure S1).

Filter influence. A small difference in sorption appears between the two filters, with slightly higher NPT sorption on the CGB-R filter, and this phenomenon was more pronounced for the 50$\mathrm{nm}$ PSL. Both filters had the same densities $\left(0.13 \mathrm{~g} / \mathrm{cm}^{3}\right)$ and the same surface-to-volume ratios (4.0). CGB-R was smaller in volume ( 0.45 and $0.75 \mathrm{~cm}^{3}$ for $C G B-R$ and $C G B-G$, respectively). These comparisons suggest that the dimensional characteristics of the filters didn't explain the influence of the filter on the sorption capacity, especially for highly diffusive species. Whatever the characteristics, both filters present high sorption capacities for all the NPTs and size ranges tested in this study.

202 It is interesting to compare the high sorption capacity for the $50-\mathrm{nm}$ nanoplastics in freshwater with the cut-off capacity observed on smoked cigarette butts. Indeed, Van Dirk et al. 
are generally transferred to humans, i.e., not retained by the cigarette filter (van Dijk et al., 2011). Other studies showed that a smoked CGB releases soot particles larger than $70 \mathrm{~nm}$ into aqueous systems (Chevalier et al., 2018). There is a fairly remarkable difference in the abilities of filters to absorb nanoparticles in water versus air.

(a)

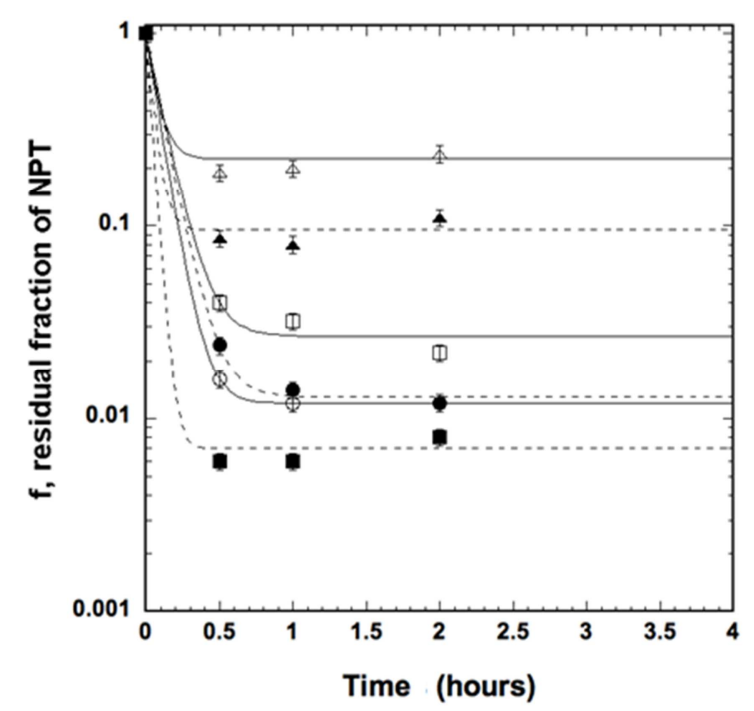

(b)

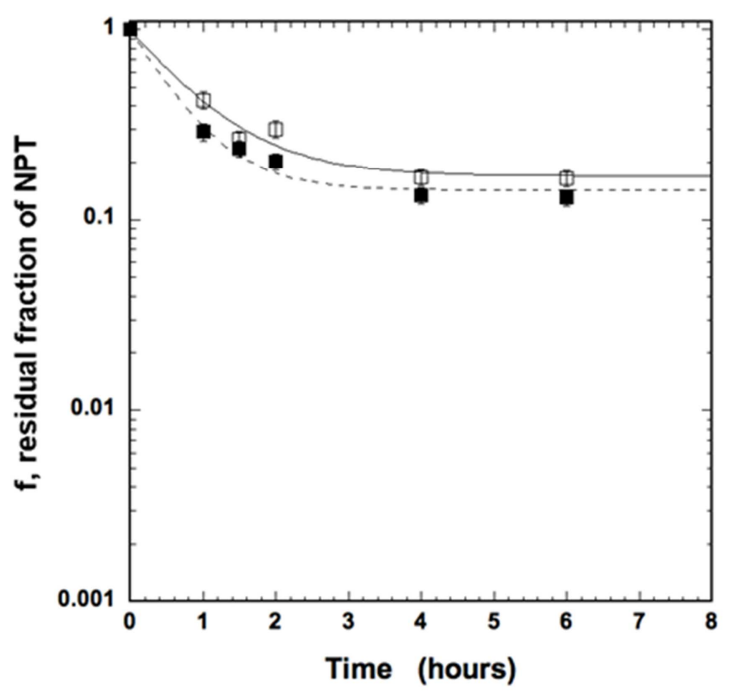

Figure 2. Sorption kinetics represented by the residual fraction $(f)$ as a function of the contact time between CGBs (R is represented by closed symbols, and G is represented by open symbols) and a) PSL NPTs (50 $\mathrm{nm}(\square \square), 180 \mathrm{~nm}(\bullet 0)$ and 500 $\mathrm{nm}(\boldsymbol{\Delta} \triangle)$ ) or b) NPTf in solution. The initial concentration of NPTs was $5 \mathrm{mg} \mathrm{L}^{-1}$. The lines correspond to the fit of the data based on the equation (2).

Sorption mechanisms. The sorption mechanisms of NPTs on carbon-based materials can be complex because electrostatic and nonelectrostatic interactions (depending on the absorbent, the absorbate and the solution chemistry) may come into play (Moreno-Castilla, 2004). Electrostatic interactions appear when charges (negative or positive) are involved. Figure 3 presents XDLVO modelling (the conditions are summarized in the SI) according to the zeta potential of cellulose acetate and the different NPT batches used in the present study (Tadros, 2013). The secondary minimum energy (insert in Figure 3) presents potentials favourable for 
reaching the filter surface for all the NPTs. The more-retained $50 \mathrm{~nm}$-PSL presents a less

223 favourable secondary energy minimum, and the less-retained $500 \mathrm{~nm}$-PSL presents a more

224 favourable minimum. Usually, the secondary minimum does not affect the sorption kinetics.

225 However, when this minimum is deep enough, especially for larger particles, initial rapid

226 attachment may occur at the level of the secondary minimum (Trefalt and Borkovec, 2014). In

227 the investigation of the primary maximum energy, this tendency corresponds to the retention

228 behaviour. The lowest energy barrier is obtained for the $50 \mathrm{~nm}$-PSL, and the highest barrier is

229 obtained for the $500 \mathrm{~nm}-\mathrm{PSL}$, which corresponds to the order experimentally observed for PSL in

230 Figure 3 and previously discussed. Under these conditions, XDLVO theory demonstrates that the

231 mechanisms allowing the retention of NPTs on the filter surface occur at a small separation

232 distance mostly for the $50 \mathrm{~nm}$-PSL, where the energy barrier is the lowest for overcoming 233 electrostatic repulsion.

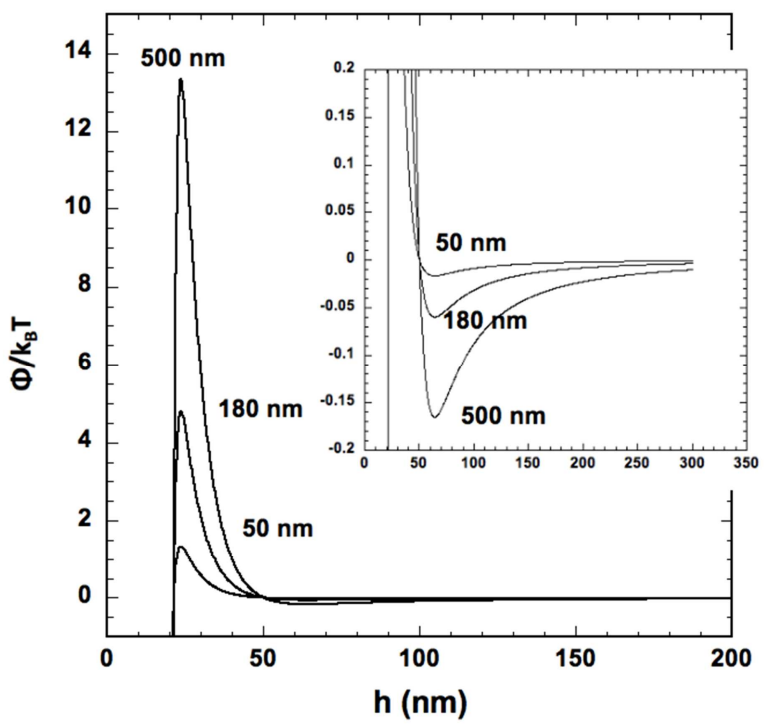

Figure 3. Potential curves of coagulation calculated by XDLVO theory for particles of various sizes.

Sorption kinetics. 
240 reports the $K_{S W}$ values determined from equation 2 . The results show partition coefficients 241 within the range of $10^{2}$ to $10^{4}$, reflecting a non-negligible bioaccumulation factor when 242 considering CGBs in the food chain. Assuming an aquatic medium contaminated with $10 \mathrm{ppb}$ of 243 NPTs, as proposed within the literature, and the 750000 metric tons of CGBs ending up as litter 244 worldwide per year (Novotny et al., 2009), 0.75 to 75 metric tons of NPTs could be absorbed by cigarette filters each year. It is worth mentioning that NPTs are only one of the contaminants 246 loaded within CGBs, as reported elsewhere (Chevalier et al., 2018). Many other contaminants, such as particles and metals, should be considered in used butts. Additionally, the partition coefficients determined for deionized water for the G-filter and 50-nm and 500-nm PSL are 8400 and 890, respectively, slightly higher than those measured in freshwater (approximately 1.8250 fold), demonstrating that the range of salinity of freshwater has little effect on the sorption of 251 NPTs, compared to the values presented above.

Table 1: water sampling rate $\mathrm{R}_{\mathrm{s}}$ and CGB-water partition coefficient $K_{s w}$ determined by adjusting the kinetic curves (Figure 2). The curve fit based on the Levenberg-Marquardt algorithm was calculated using an iterative procedure on equation 2.

\begin{tabular}{|c|c|c|c|c|c|}
\hline \multirow{2}{*}{ Sample } & \multirow{2}{*}{$\begin{array}{c}\text { z-average } \\
(\mathrm{nm})\end{array}$} & \multicolumn{2}{|c|}{$R_{s}(m L / m i n)$} & \multicolumn{2}{|c|}{$\mathrm{K}_{\mathrm{sw}}$} \\
\hline & & G & $\mathrm{R}$ & G & $\mathrm{R}$ \\
\hline PSL & 50 & 96 & $100 *$ & 4450 & $24000^{*}$ \\
\hline PSL & 180 & 108 & 175 & 12370 & 16600 \\
\hline PSL & 500 & 107 & 150 & 520 & 1840 \\
\hline NPTf & 310 & 10 & 17 & 710 & 1380 \\
\hline
\end{tabular}

In Table 1, the differences between the $R_{s}$ values obtained for CGB-G and CGB-R are due to the calculation method (see Equation (2)) based only on the CGB external dimensions and not the porosity. The surface area ratio between CGB-G and CGB-R is 0.7 , while the corresponding $R_{s}$ 
ratio is $0.6-0.7$. Furthermore, the $R_{s}$ values are significantly different for PSLs and NPTf, with a

266 value one order of magnitude lower for the latter. All other things being equal, $R_{s}$ depends on

267 the diffusion coefficient of NPTs in CGB through the overall mass transfer coefficient, $k_{0}$. The

268 NPT $f$ sample has an intermediate diffusion coefficient compared to those of 180-nm PSL and

269 500-nm PSL, as shown by the results reported in Figure 2. Based on these results, the filter

270 nature (i.e. $\mathrm{R}$ or $\mathrm{G}$ ) has negligible contribution to sorption capacity rather than the size and

271 morphology of nanoplastics. More specifically, the anisotropy of the NPT $f$ sample may be what

272 strongly affects its diffusion coefficient in CGBs.

273 Unfortunately, quantitative experiments on used butts could not be carried out with the 274 analytical methodology proposed in this work. Used CGBs release organic matter, including a 275 nanoparticulate fraction that strongly interferes with the results of AF4 analysis. However, from 276 a qualitative point of view and as illustrated within the graphical abstract, a 20-ppm water 277 solution of blue coloured PSL $200 \mathrm{~nm}$ quickly turns colourless when either an unused or used 278 CGB is added. The exposure concentrations and times are similar to those of the experiment 279 using an unused CGB as described above. This qualitative result reinforces the idea of similar 280 strong NPT sorption on both unused and used CGBs.

\section{Conclusions}

283 In this work, we addressed the problem of the behaviour of nanoplastics (NPTs) in the 284 environment through a better understanding of their interaction with one of the most 285 predominant types of waste: cigarette butts. We also demonstrated the high capacity of NPTs to 286 be absorbed on cigarette butts. A quantitative kinetic approach was used to evaluate the 287 partition coefficients between NPTs and CGBs, which ranged from $10^{2}$ to $10^{4}$ at room 
environment may be transferred to porous organic matrices. The determination of the water sampling rate demonstrates the significant effect of nanoplastic morphology on the sorption behaviour. Nanoplastics prepared by mechanical fragmentation with high size polydispersity and anisotropic morphology exhibit diffusion coefficients in butts that differ from those obtained for

NPTs with spherical morphologies such as PSLs. This result also demonstrates the importance of

taking into consideration the morphology of nanoplastic samples when determining the mechanisms that govern the transfer, accumulation and fate of NPTs.

\section{Conflicts of interest}

There are no conflicts of interest to declare.

\section{Acknowledgements}

This research was carried out under the framework of E2S UPPA supported by the

"Investissements d'Avenir" French programme managed by ANR (ANR-16-IDEX-0002).

301

302

\section{Notes and references}

Alimi, O.S., Farner Budarz, J., Hernandez, L.M., Tufenkji, N., 2018. Microplastics and Nanoplastics in Aquatic Environments: Aggregation, Deposition, and Enhanced Contaminant Transport. Environ. Sci. Technol. 52, 1704-1724. https://doi.org/10.1021/acs.est.7b05559

Araújo, M.C.B., Costa, M.F., 2019. A critical review of the issue of cigarette butt pollution in coastal environments. Environ. Res. 172, 137-149. https://doi.org/10.1016/j.envres.2019.02.005

Brewer, A.K., Striegel, A.M., 2011. Characterizing String-of-Pearls Colloidal Silica by Multidetector Hydrodynamic Chromatography and Comparison to Multidetector Size-Exclusion Chromatography, Off-Line Multiangle Static Light Scattering, and Transmission Electron Microscopy. Anal. Chem. 83, 3068-3075. https://doi.org/10.1021/ac103314c

Chevalier, Q., El Hadri, H., Petitjean, P., Bouhnik-Le Coz, M., Reynaud, S., Grassl, B., Gigault, J., 2018. Nano-litter from cigarette butts: Environmental implications and urgent consideration. Chemosphere 194, 125-130. https://doi.org/10.1016/j.chemosphere.2017.11.158

da Costa, J.P., Santos, P.S.M., Duarte, A.C., Rocha-Santos, T., 2016. (Nano)plastics in the environment Sources, fates and effects. Sci. Total Environ. 566-567, 15-26. https://doi.org/10.1016/j.scitotenv.2016.05.041 
Davranche, M., Veclin, C., Pierson-Wickmann, A.-C., El Hadri, H., Grassl, B., Rowenczyk, L., Dia, A., Ter Halle, A., Blancho, F., Reynaud, S., Gigault, J., 2019. Are nanoplastics able to bind significant amount of metals? The lead example. Environ. Pollut. 249, 940-948. https://doi.org/10.1016/j.envpol.2019.03.087

Dong, Z., Zhu, L., Zhang, W., Huang, R., Lv, X., Jing, X., Yang, Z., Wang, J., Qiu, Y., 2019. Role of surface functionalities of nanoplastics on their transport in seawater-saturated sea sand. Environ. Pollut. 255, 113177. https://doi.org/10.1016/j.envpol.2019.113177

El Hadri, H., Gigault, J., Maxit, B., Grassl, B., Reynaud, S., 2020. Nanoplastic from mechanically degraded primary and secondary microplastics for environmental assessments. Nanolmpact 17. https://doi.org/10.1016/j.impact.2019.100206

Gigault, J., El, H., Reynaud, S., Deniau, E., Grassl, B., 2017. Asymmetrical flow field flow fractionation methods to characterize submicron particles: application to carbon-based aggregates and nanoplastics. Anal. Bioanal. Chem. 409, 6761-6769. https://doi.org/10.1007/s00216-017-0629-7 Gigault, J., Pedrono, B., Maxit, B., Halle, A.T., 2016. Marine plastic litter: the unanalyzed nano-fraction. Environ. Sci.: Nano 3, 346-350. https://doi.org/10.1039/C6EN00008H

Gillibert, R., Balakrishnan, G., Deshoules, Q., Tardivel, M., Magazzù, A., Donato, M.G., Maragò, O.M., Lamy de La Chapelle, M., Colas, F., Lagarde, F., Gucciardi, P.G., 2019. Raman Tweezers for Small Microplastics and Nanoplastics Identification in Seawater. Environ. Sci. Technol. 53, 9003-9013. https://doi.org/10.1021/acs.est.9b03105

Hartmann, N.B., Hüffer, T., Thompson, R.C., Hassellöv, M., Verschoor, A., Daugaard, A.E., Rist, S., Karlsson, T., Brennholt, N., Cole, M., Herrling, M.P., Hess, M.C., Ivleva, N.P., Lusher, A.L., Wagner, M., 2019. Are We Speaking the Same Language? Recommendations for a Definition and Categorization Framework for Plastic Debris. Environ. Sci. Technol. 53, 1039-1047. https://doi.org/10.1021/acs.est.8b05297

Hengstberger, M., Stark, M., 2009. Fibre and Particle Release from Cigarette Filters. Beitr. Zur Tab. Int. Tob. Res. 23, 338-358. https://doi.org/10.2478/cttr-2013-0869

Lambert, S., Wagner, M., 2016a. Characterisation of nanoplastics during the degradation of polystyrene. Chemosphere 145, 265-268. https://doi.org/10.1016/j.chemosphere.2015.11.078

Lambert, S., Wagner, M., 2016b. Formation of microscopic particles during the degradation of different polymers. Chemosphere 161, 510-517. https://doi.org/10.1016/j.chemosphere.2016.07.042

Li, P., Li, Q., Hao, Z., Yu, S., Liu, J., 2020. Analytical methods and environmental processes of nanoplastics. J. Environ. Sci. 94, 88-99. https://doi.org/10.1016/j.jes.2020.03.057

Mackay, J., Eriksen, M., Eriksen, M.P., 2002. The tobacco atlas. World Health Organization.

Moreno-Castilla, C., 2004. Adsorption of organic molecules from aqueous solutions on carbon materials. Carbon 42, 83-94. https://doi.org/10.1016/j.carbon.2003.09.022

Novotny, T., Lum, K., Smith, E., Wang, V., Barnes, R., 2009. Cigarettes Butts and the Case for an Environmental Policy on Hazardous Cigarette Waste. Int. J. Environ. Res. Public. Health 6, 16911705. https://doi.org/10.3390/ijerph6051691

Novotny, T.E., Slaughter, E., 2014. Tobacco Product Waste: An Environmental Approach to Reduce Tobacco Consumption. Curr. Environ. Health Rep. 1, 208-216. https://doi.org/10.1007/s40572014-0016-x

Pessoni, L., Veclin, C., El Hadri, H., Cugnet, C., Davranche, M., Pierson-Wickmann, A.-C., Gigault, J., Grassl, B., Reynaud, S. (2019) Soap- and metal-free polystyrene latex particles as a nanoplastic model. Environmental Science: Nano. 6, 2253-2258.

Pikuda, O., Xu, E.G., Berk, D., Tufenkji, N., 2018. Toxicity Assessments of Micro- and Nanoplastics Can Be Confounded by Preservatives in Commercial Formulations. Environ. Sci. Technol. Lett. 6, 21-25. https://doi.org/10.1021/acs.estlett.8b00614 
Pu, D., Kou, Y., Zhang, L., Liu, B., Zhu, W., Zhu, L., Duan, T., 2019. Waste cigarette filters: activated carbon as a novel sorbent for uranium removal. J. Radioanal. Nucl. Chem. 320, 725-731. https://doi.org/10.1007/s10967-019-06502-z

Schmitt, C., Grassl, B., Lespes, G., Desbrières, J., Pellerin, V., Reynaud, S., Gigault, J., Hackley, V.A., 2014. Saponins: A Renewable and Biodegradable Surfactant From Its Microwave-Assisted Extraction to the Synthesis of Monodisperse Lattices. Biomacromolecules 140131113511009. https://doi.org/10.1021/bm401708m

Shen, M., Zhang, Y., Zhu, Y., Song, B., Zeng, G., Hu, D., Wen, X., Ren, X., 2019. Recent advances in toxicological research of nanoplastics in the environment: A review. Environ. Pollut. 252, 511521.

Tadros, T., 2013. Elastic Repulsion, in: Tadros, T. (Ed.), Encyclopedia of Colloid and Interface Science. Springer Berlin Heidelberg, Berlin, Heidelberg, pp. 341-341. https://doi.org/10.1007/978-3-64220665-8_73

Trefalt, G., Borkovec, M., 2014. Overview of DLVO theory. Lab. Colloid Surf. Chem. Univ. Geneva 29. van Dijk, W.D., Gopal, S., Scheepers, P.T.J., 2011. Nanoparticles in cigarette smoke; real-time undiluted measurements by a scanning mobility particle sizer. Anal. Bioanal. Chem. 399, 3573-3578. https://doi.org/10.1007/s00216-011-4701-4

Wagner, S., Reemtsma, T., 2019. Things we know and don't know about nanoplastic in the environment. Nat. Nanotechnol. 14, 300-301. https://doi.org/10.1038/s41565-019-0424-z 
Fate of nanoplastics in the environment: implication of the cigarette butts

Hind El Hadri, Jesus Maza Lisa, Julien Gigault, Stéphanie Reynaud, Bruno Grassl

\section{Highlights:}

- The nanoplastic sorption by cigarette filters was evaluated in pure and freshwater

- The sorption was addressed for nanoplastics of various morphologies and dispersity.

- A kinetic approach gives cigarette filters partition coefficient within $10^{2}-10^{4}$ 


\section{Author statements}

Dr. Hind El Hadri: methodology, investigation, writing

Dr. Jesus Maza-Lisa: methodology, investigation

Dr. Julien Gigault: Supervision, investigation, writing

Dr. Stéphanie Reynaud: Supervision, Resources, writing

Prof Bruno Grassl: Supervision, Resources, writing 